\title{
Bilateral Diffuse Uveal Melanocytic Proliferation Presenting as a Giant Unilateral Choroidal Nevus: A Case Report
}

\author{
Carlos Menezes $^{a}$ Rui Carvalho ${ }^{a} \quad$ Joana Neves-Martins ${ }^{a-c} \quad$ Carla Teixeira $^{a}$ \\ ${ }^{a}$ Department of Ophthalmology, Hospital Pedro Hispano, Matosinhos, ${ }^{b}$ Clínica Oftalmológica Dr. Neves Martins, \\ Porto, and ${ }^{\mathrm{C} H o s p i t a l}$ Privado da Trofa, Trofa, Portugal
}

\section{Key Words}

Bilateral diffuse uveal melanocytic proliferation .

Paraneoplastic retinopathy . Chemotherapy

\begin{abstract}
Background/Aims: The aim of our study was to report a case of bilateral diffuse uveal melanocytic proliferation (BDUMP) with a markedly asymmetric presentation and fundoscopic response to palliative chemotherapy. Case Report: We report a 67-year-old Caucasian man who presented with vision loss in his right eye. The best-corrected visual acuities were $2 / 10$ in the right eye and 10/10 in the left eye, and biomicroscopy revealed bilateral mild cataracts. Fundoscopy of the right eye showed a macular flat and pigmented lesion extending beyond the posterior pole with areas of giraffe-type pigmentation and an overlying exudative retinal detachment. Nothing remarkable was detected in the left eye apart from a small round hypopigmented area of retinal pigment epithelium atrophy in the papillomacular bundle. BDUMP was diagnosed, and the workup for systemic malignancy revealed a pulmonary adenocarcinoma. After chemotherapy, not only did the right eye's visual acuity improve and the
\end{abstract}

\section{KARGER}

(c) 2015 S. Karger AG, Basel

2296-4681/15/0021-0054\$39.50/0

E-Mail karger@karger.com

www.karger.com/oop serous detachment resolve, but also the pigmentation decreased. Conclusion: BDUMP presentation can be markedly asymmetric and resemble a giant unilateral choroidal nevus. Response to chemotherapy was unique not only for the usual retinal detachment resolution, but also because of an evident regression of pigmentation.

(c) 2015 S. Karger AG, Basel

\section{Introduction}

Bilateral diffuse uveal melanocytic proliferation (BDUMP) is a rare paraneoplastic syndrome, with a poor general and visual prognosis. It was first described by Machemer [1] in 1966 and named by Barr et al. [2] in 1982. Its five main diagnostic characteristics were described by Gass et al. [3] in 1990, namely: (1) elevated polygonal red patches at the level of the retinal pigment epithelium (RPE) with (2) associated early hyperfluorescence, (3) slightly elevated uveal melanocytic tumors with a variable degree of pigmentation or diffuse uveal thickening, (4) exudative retinal detachment and (5) rapidly progressing cataracts. Gass et al. [3] described the first two findings as early fea- 


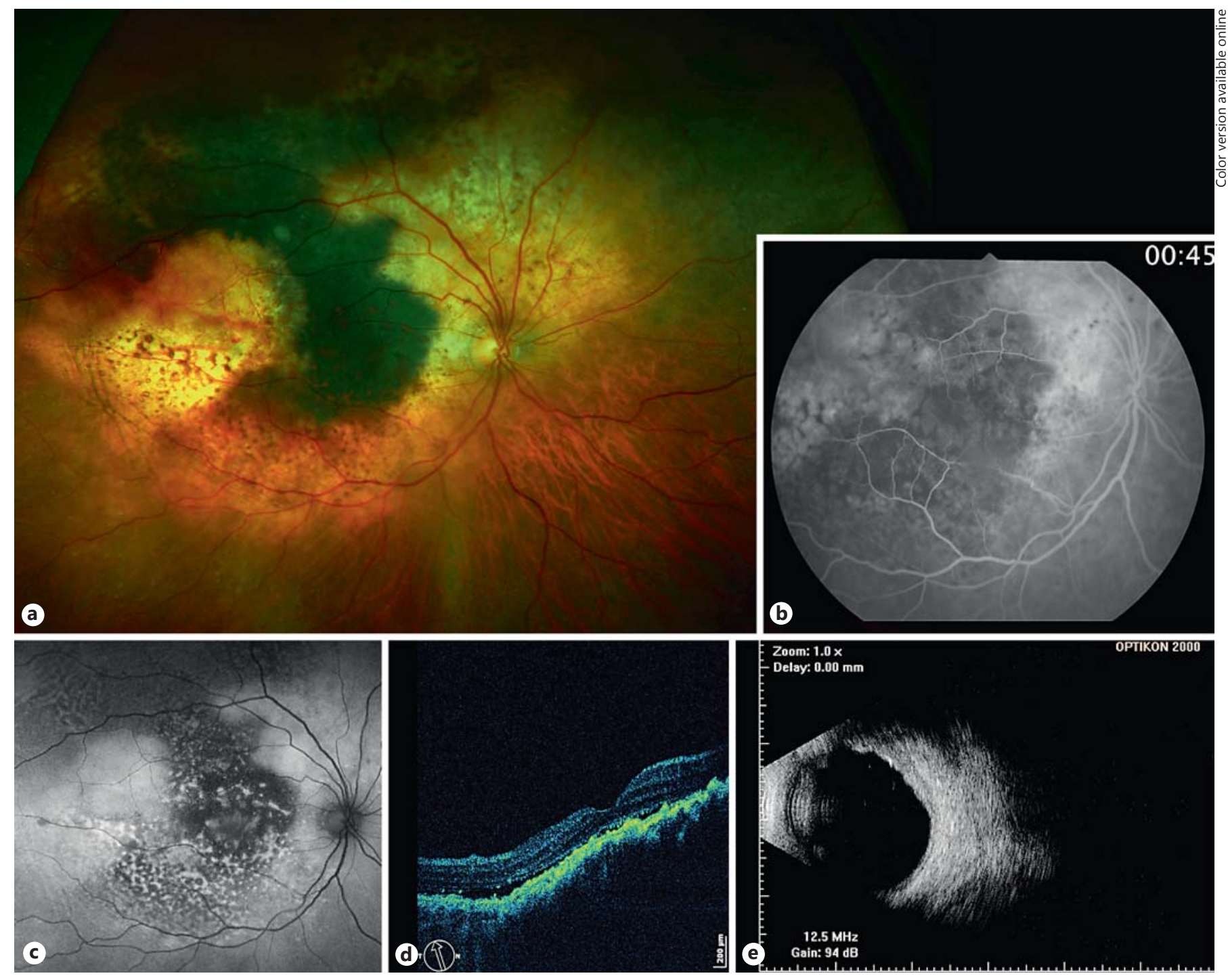

Fig. 1. Multimodal imaging of the OD retina before chemotherapy. a Wide-angle color fundus photography of the OD showing a dark pigmented macular lesion extending superiorly and temporally, with areas of orange giraffe-type pigmentation, areas of RPE atrophy and an exudative retinal detachment that extends superiorly and nasally beyond the posterior pole. b Fluorescein angiography of the OD showing blocking defects by the pigmented choroidal lesion as well as hyperfluorescent areas due to RPE atrophy within hypofluorescent polygonal limits due to segregation of RPE. c Fundus autofluorescence of the OD showing increased autofluorescence of the aggregation RPE areas due to accumulation of lipofuscin and loss of autofluorescence in areas of RPE atrophy. $\mathbf{d}$ OCT of the OD showing a foveal neurosensorial detachment and areas of complete loss of both the RPE and photoreceptors with an adjacent thickened RPE in areas of RPE aggregation. e B-scan ocular ultrasound of the OD showing diffuse superior choroidal thickening. tures and the last three as late features of the disease, the latter being associated with a very poor prognosis.

Findings and symptoms are typically bilateral, and treatment is mainly ineffective. We report an exceptional case for its asymmetry and fundoscopic response to chemotherapy - a rare presentation and evolution of the disease.

\section{Case Presentation}

A 67-year-old man presented with progressive loss of vision in the right eye (OD) for 6 months. He was a heavy smoker and reported constitutional symptoms. He had had an ophthalmologic examination 3 years before, which was described as normal.

The best-corrected visual acuities were $2 / 10$ in the OD and $10 / 10$ in the left eye (OS). Biomicroscopy revealed bilateral mild 

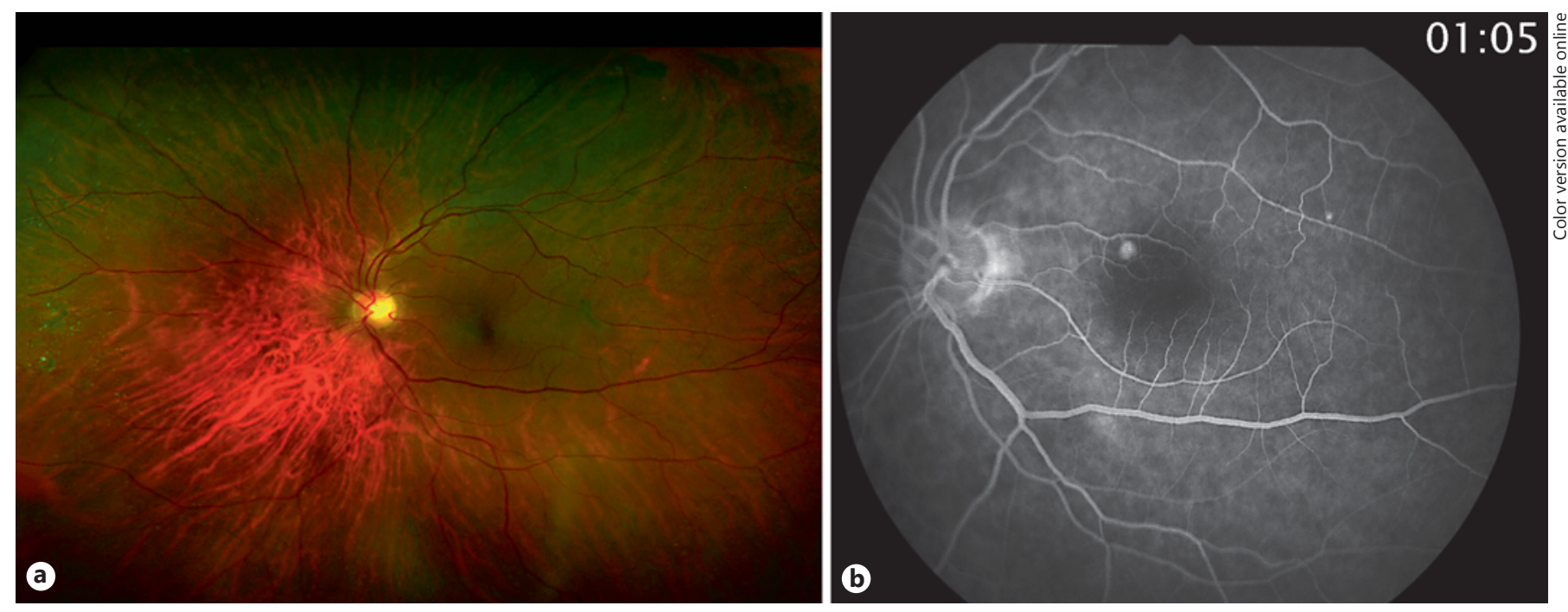

Fig. 2. Multimodal imaging of the OS retina before chemotherapy. a Wide-angle color fundus photography of the OS showing a normal fundus, with the exception of a small round hypopigmented area in the papillomacular bundle. b Fluorescein angiography of the OS showing two round patches of early hyperfluorescence. cataracts, and the intraocular pressures were 16/17 mm Hg. No cells in the anterior chamber or vitreous were present. There was no abnormal pigmentation of the external ocular structures either.

Ophthalmoscopy showed a slightly elevated and pigmented macular lesion in the OD extending beyond the posterior pole, resembling a giant choroidal nevus, with areas of giraffe-type pigmentation and a thin but extensive exudative retinal detachment with macular commitment (fig. 1a). Fluorescein angiography revealed blocking by the hyperpigmented lesion and hyperfluorescent areas due to RPE atrophy within hypofluorescent areas due to the segregation of RPE (fig. 1b). This pattern was inverse in the fundus autofluorescence (fig. 1c). Spectral-domain optical coherence tomography (OCT; Copernicus; Optopol) showed a macular neurosensorial detachment and the alterations responsible for the 'giraffe sign' pattern, namely areas of complete loss of both the RPE and the ellipsoid line with an adjacent thickened RPE in areas of RPE aggregation (fig. 1d). A diffuse choroidal thickening was evident on B-scan ultrasonography (fig. 1e).

Nothing remarkable was detected in the OS, apart from a small round hypopigmented area in the papillomacular bundle (fig. 2a). Fluorescein angiography showed two round patches of early hyperfluorescence, one corresponding to the described lesion, and the other smaller, temporal to the fovea and not visible in the fundoscopy (fig. 2b). OCT highlighted the first finding as a round area of RPE atrophy.

BDUMP was diagnosed and the systemic study revealed an unresectable pulmonary adenocarcinoma with adrenal gland and multiple bone metastasis - stage IV (cT4NOM1). The patient underwent 6 cycles of palliate chemotherapy with carboplatin and pemetrexed, combined with zoledronate, with an optimal response, namely an improvement in functional status and partial tumor regression.

Seven months after the diagnosis, when chemotherapy was finished, BCVA were $3 / 10$ in the OD and 10/10 in the OS. OD lesions partially regressed, with a decrease in pigmentation (fig. 3a, b), resolution of the exudative detachment (fig. 3c) and normalization of choroidal thickness (fig. 3d). The OS remained stable. One year after the diagnosis the patient died due to cancer complications.

\section{Discussion}

BDUMP is characterized by the proliferation of spindle-shaped melanocytes within the choroid and consequent diffuse uveal thickening [4]. In recent years, numerous new case reports have increased our knowledge of BDUMP - its nature, findings and treatment. Additional associated findings have been described, namely iris nodules [5], pigmented keratic precipitates, anterior chamber and vitreous cells [4], peripheral retinal arterial nonperfusion [6], loss of RPE in a pattern described as nummular [5] or dermal and conjunctival melanocytic proliferation [7].

This case is unique for its asymmetry and presentation as a giant choroidal flat nevus. Although both a choroidal melanoma and a choroidal metastasis from the primary pulmonary adenocarcinoma were important differential diagnoses, the clinical findings of the OD were typical of BDUMP. While the OD fit all cardinal signs [3] except a significant cataract, the OS had only features of early disease. A unilateral case of BDUMP was reported by Reddy and Finger [8] in a woman with an ipsilateral choroidal metastasis from a small cell lung carcinoma. The authors 

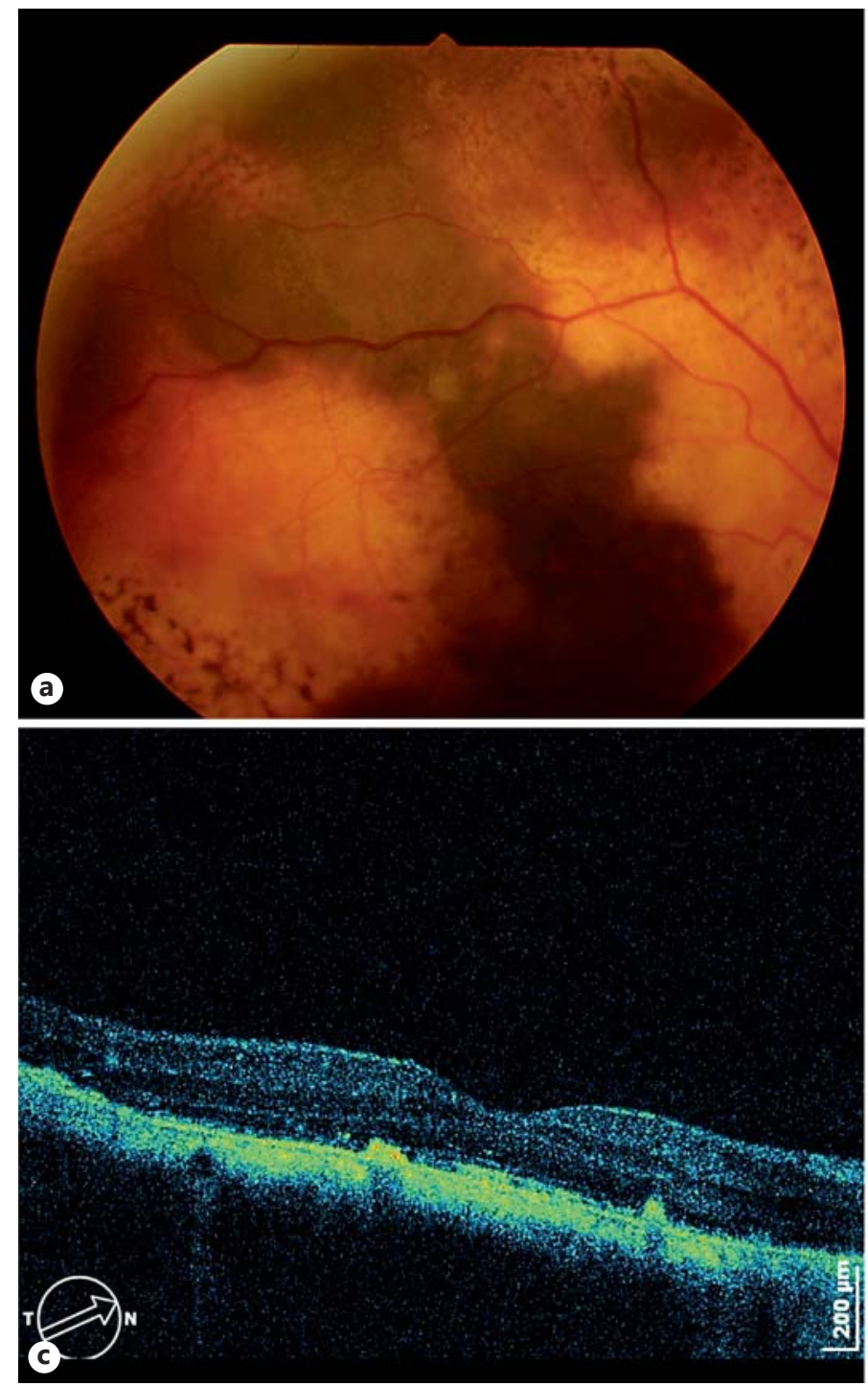

Fig. 3. Multimodal imaging of the OD retina after chemotherapy. a, b Color fundus photography showing an evident decrease in the lesion's pigmentation, most evident in the upper limit of the lesion.

hypothesized the local production of growth factors by the metastasis as the cause. Other asymmetric presentations of BDUMP have also been reported. Borruat et al. [9] described a patient who presented with unilateral multiple typical lesions of BDUMP before bilateral involvement, and other authors reported initial lesions that mimicked small unilateral choroidal melanomas $[10,11]$. Our patient, however, presented with a unilateral much more evolved lesion that we believe is the largest isolated flat nevus ever described in the context of BDUMP and, perhaps, one of the most premature lesions in the fellow eye.
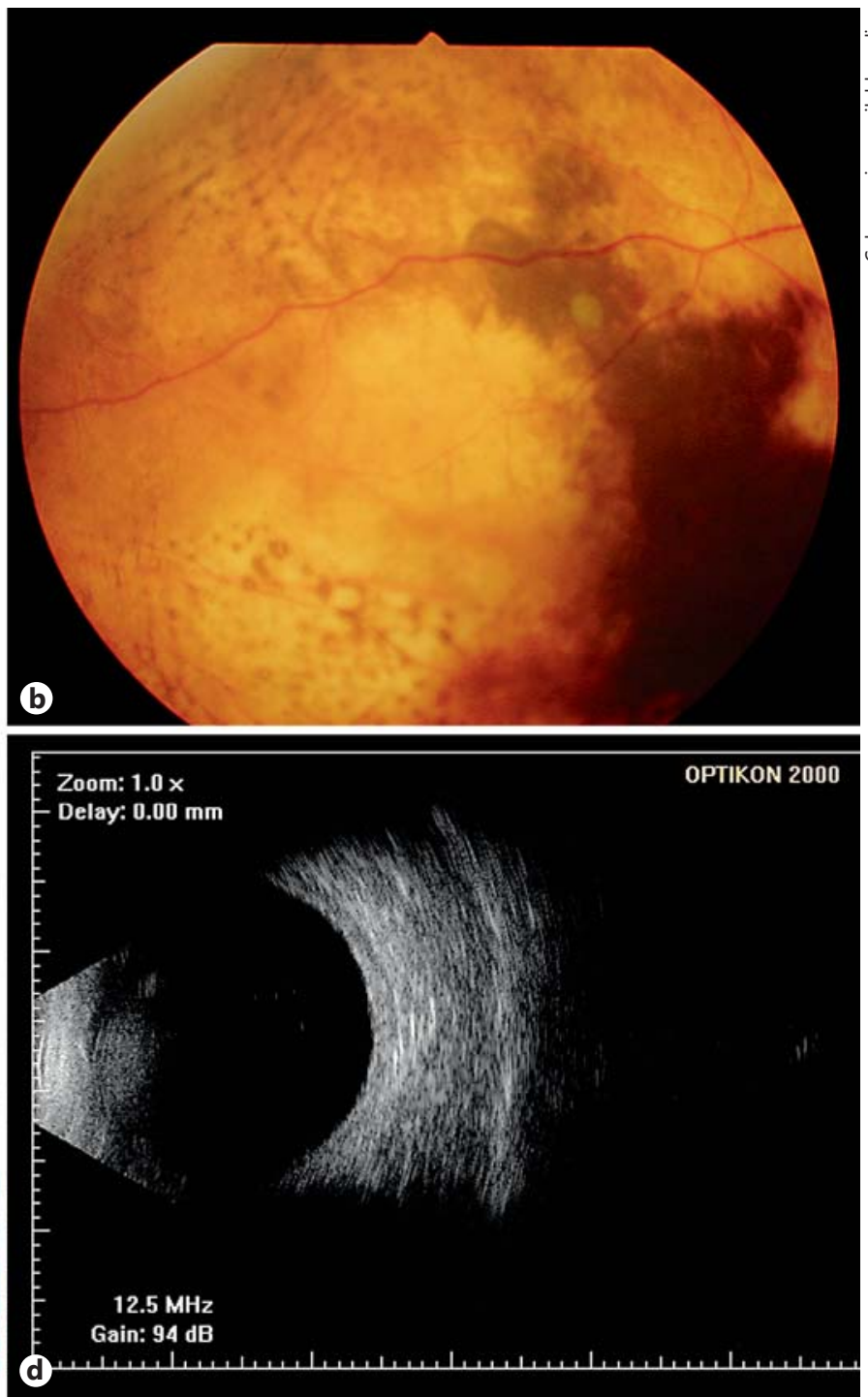

c OCT showing resolution of the serous detachment as well as decrease in RPE irregularity. d B-scan ocular ultrasound showing normalization of choroidal thickening.

Apart from the recently recognized potential of plasmapheresis to stabilize vision in patients with BDUMP $[12,13]$, treatment has until now mainly been ineffective $[4,14]$. Corticosteroids, radiation and drainage of subretinal fluid all failed to give consistent results [5]. Treatment aimed at the primary tumor has also shown variable results. A single case of a curative resection of the primary tumor was reported by Sen et al. [15], though too late to preserve useful vision. Our case shows the regression of BDUMP lesions and improvement in visual acuity after a well-succeeded palliative chemotherapy. 
These facts enhance the paraneoplastic nature of this entity and prove that, when effective, the primary tumor regression is accompanied by the regression of BDUMP lesions. In our case, not only did the serous detachment and choroidal thickness normalize, but also the choroid pigmentation markedly decreased in an unparalleled way. Despite this good response, as in the majority of patients with BDUMP, our patient died within 1 year after the onset of the disease [14].

\section{Conclusion}

BDUMP presentation can be markedly asymmetric and resemble a giant unilateral choroidal nevus. An evident regression of pigmentation accompanying exudative retinal detachment resolution may occur as part of a well-succeeded response to chemotherapy.

\section{Acknowledgments}

The authors thank Dr. Miguel Abreu, MD (Department of Oncology, Portuguese Institute of Oncology, Porto, Portugal), for his important contribution to the acquisition, analysis and interpretation of the data of this work, and Dr. José Alberto Lemos (Department of Ophthalmology, Pedro Hispano Hospital, Matosinhos, Portugal) for his contribution to the analysis and interpretation of the data of this work.

\section{Statement of Ethics}

Informed consent was obtained from all authors and from the patient's family before submitting this article and accompanying images for publication. A copy of the written consent is available for review from the Editor-in-Chief of this journal.

\section{Disclosure Statement}

The authors declare that they have no conflict of interest.

\section{References}

1 Machemer R: Zur Pathogenese des flächenhaften malignen Melanoms. Klin Monatsbl Augenheilkd 1966;149:641-652.

2 Barr CC, Zimmerman LE, Curtin VT, Font RL: Bilateral diffuse melanocytic uveal tumours associated with systemic malignant neoplasms: a recently recognized syndrome. Arch Opthalmol 1982;100:249-255.

3 Gass JD, Gieser RG, Wilkinson CP, Beahm DE, Pautler SE: Bilateral diffuse uveal melanocytic proliferation in patients with occult carcinoma. Arch Ophthalmol 1990;108:527533.

4 Lin P, Mruthyunjaya P: Retinal manifestations of oncologic and hematologic diseases. Int Ophthalmol Clin 2012;52:67-91.

5 Navajas EV, Simpson ER, Krema H, Hammoudi DS, Weisbrod D, Bernardini M, Altomare F: Cancer-associated nummular loss of RPE: expanding the spectrum of bilateral diffuse uveal melanocytic proliferation. Ophthalmic Surg Lasers Imaging 2011;42:e103e106.
6 6 Yonekawa Y, Shidkrot Y, Eliott D: Inferior peripheral non-perfusion in bilateral diffuse uveal melanocytic proliferation. Ophthalmic Surg Lasers Imaging Retina DOI: 10.3928/ 23258160-20130313-11.

7 Pulido JS, Flotte TJ, Raja H, Miles S, Winters JL, Niles R, Jaben EA, Markovic SN, Davies J, Kalli KR, Vile RG, Garcia JJ, Salomao Dr: Dermal and conjunctival melanocytic proliferations in diffuse uveal melanocytic proliferation. Eye (Lond) DOI: 10.1038/eye.2013.131.

8 Reddy S, Finger PT: Unilateral diffuse uveal melanocytic proliferation (DUMP). Br J Ophthalmol 2007;91:1726-1727.

-9 Borruat FX, Othenin-Girard P, Uffer S, Othenin-Girard B, Regli F, Hurlimann J: Natural history of diffuse uveal melanocytic proliferation. Case report. Ophthalmology 1992; 99:1698-1704.

10 Ulrich JN, Garg S, Escaravage GK, Meredith TM: Bilateral diffuse uveal melanocytic proliferation presenting as small choroidal melanoma. Case Rep Ophthalmol Med DOI: 10.1155/2011/740640.

11 Donovan JT, Prefontaine M, Gragoudas ES: Blindness as a consequence of a paraneoplastic syndrome in a woman with clear cell carcinoma of the ovary. Gynecol Oncol 1999;73: 424-429.
12 Jaben EA, Pulido JS, Pittock S, Markovic S, Winters JL: The potential role of plasma exchange as a treatment for bilateral diffuse uveal melanocytic proliferation: a report of two cases. J Clin Apher 2011;26:356-336.

13 Mets RB, Golchet P, Adamus G, Anitori R, Wilson D, Shaw J, Jampol LM: Bilateral diffuse uveal melanocytic proliferation with a positive ophthalmoscopic and visual response to plasmapheresis. Arch Ophthalmol DOI: 10.1001/archophthalmol.2011.277.

14 Rahimy E, Sarraf D: Paraneoplastic and nonparaneoplastic retinopathy and optic neuropathy: evaluation and management. Surv Ophtalmol DOI: 10.1016/j.survophthal.2012.09.001.

15 Sen J, Clewes A, Quah S, Hiscott P, Bucknall R, Damato B: Presymptomatic diagnosis of bronchogenic carcinoma associated with bilateral diffuse uveal melanocytic proliferation. Clin Experiment Ophthalmol DOI: 10.1111/j.1442-9071.2006.1145.x. 